\title{
ACAROLOGY
}

\section{Plano de Amostragem Presença-Ausência para Oligonychus yothersi (McGregor) (Acari: Tetranychidae) na Cultura da Erva-Mate}

\author{
Alfredo de Gouvea ${ }^{1}$ Guilherme Bertoldo $^{2}$ e Luis F.A. Alves ${ }^{3}$ \\ ${ }^{1}$ Universidade Tecnológica Federal do Paraná - Campus Dois Vizinhos, Estrada para Boa Esperança, km 4 \\ 85660-000, Dois Vizinhos, PR \\ ${ }^{2}$ Depto. Física, Univ. Federal do Paraná, CEP 81531-990, Curitiba, PR \\ ${ }^{3}$ Lab. Zoologia, UNIOESTE/CCBS, Campus de Cascavel, PR, bolsista de Produtividade em Pesquisa
}

Neotropical Entomology 36(4):583-586 (2007)

\begin{abstract}
Presence-Absence Sampling Plan for Oligonychus yothersi (McGregor) (Acari: Tetranychidae) on Mate-Tea Orchard
\end{abstract}

\begin{abstract}
Mite infestations to the culture of mate-tea frequently causes losses by the premature fall of the leaves. So, it is necessary to monitor the population of these arthropods, and to adopt management strategies for their control. The objective of this research was to evaluate the trustworthiness of presenceabsence sampling for Oligonychus yothersi (McGregor) on mate-tea orchard. This study was conducted in Cascavel, Paraná State, from April 2001 to July 2000, in a mate-tea tree commercial plantation. Biweekly sampling of 240 leaves were collected in different parts of 10 plants, and the number of mites was counted. The aggregation pattern was determined through the coefficients $a$ and $b$ of Taylor's power law. The proportion of infested leaves and the number of required samples were estimated through mathematical model. The mite $O$. yothersi presented aggregate distribution. The proportion of infested leaves calculated by means of mathematical model showed to be a trustworthy parameter to estimate the population density of the mite. The number of required samples was small, making feasible the practical application of the presence-absence sampling method for O. yothersi in the culture of mate-tea.
\end{abstract}

KEY WORDS: Binomial sampling, red mite, purple mite, integrated pest management, Ilex paraguariensis

RESUMO - O ataque de ácaros na cultura da erva-mate tem freqüentemente provocado perdas pela queda precoce das folhas. Assim, há necessidade de se monitorar a população desses artrópodes e adotar estratégias de manejo. O objetivo deste trabalho foi avaliar a confiabilidade da amostragem presença-ausência para Oligonychus yothersi (McGregor) na erva-mate. O trabalho foi realizado em um erval comercial, em Cascavel, PR, de abril de 1999 a julho de 2000. Coletas quinzenais de 240 folhas foram feitas em diferentes partes de 10 plantas escolhidas ao acaso. A contagem dos ácaros nas folhas foi realizada em laboratório com auxílio de microscópio estereoscópico. Foi determinado o padrão de agregação através dos coeficientes a e $\mathrm{b}$ da lei da potência de Taylor; a proporção de folhas infestadas e o número de amostras requeridas foram estimados através de modelo matemático. $O$. yothersi apresentou distribuição agregada. A proporção de folhas infestadas calculada pelo modelo matemático mostrou ser um parâmetro confiável para estimar a densidade populacional do ácaro. O número de amostras requeridas foi pequeno, viabilizando a aplicação prática do método de amostragem presença-ausência para O. yothersi na cultura da erva-mate.

PALAVRAS-CHAVE: Amostragem binomial, ácaro-vermelho, ácaro-roxo, manejo integrado de pragas, Ilex paraguariensis

Com a devastação das matas de araucária, as áreas de exploração da erva-mate se reduziram e conseqüente diminuiu a oferta de matéria-prima para a indústria ervateira. Frente à necessidade de atender à crescente demanda do mercado, foi estimulado o plantio de ervais em monocultivo. A formação de povoamentos puros, além de provocar alterações fisiomorfológicas nas plantas, interferiu na complexa cadeia alimentar, acarretando rápido aumento populacional de muitas espécies fitófagas (Penteado 1995, Gouvea et al. 2006). Esse foi o caso dos ácaros, resultando em perdas na produção, pela queda precoce de folhas e secamento de brotações (Alves et al. 2004). Entre os ácaros está Oligonychus yothersi (McGregor), praga também conhecida por ácaro-roxo ou ácaro-vermelho da erva-mate.

Aumentos na densidade populacional e nos danos causados por esse ácaro, em determinados períodos e 
locais, sugere a necessidade de conduzir estudos para obter informações sobre o seu comportamento, tendo em vista o seu manejo. O levantamento populacional de organismos pequenos e abundantes, caso de ácaros, demanda excesso de tempo e pode resultar em estimativa imprecisa, de difícil aplicação prática (Ribeiro et al. 1990, Perruso \& Cassino 1997). Uma das alternativas para solucionar esse problema é a amostragem pelo método presença-ausência (binomial), que considera se a espécie está presente ou não, independentemente do número de indivíduos (Wilson \& Room 1983).

O presente trabalho teve por objetivo avaliar a confiabilidade do método de amostragem presença-ausência para $O$. yothersi, visando contribuir na implementação do Manejo Integrado de Pragas na cultura de erva-mate.

\section{Material e Métodos}

O estudo foi realizado em um erval comercial não tratado com produtos fitossanitários, conduzido em monocultivo, formadas por erveiras com aproximadamente 10 anos, arranjadas no espaçamento de 4 x 1,5 m, situado no município de Cascavel, região Oeste do Paraná.

As amostragens foram realizadas quinzenalmente, de abril de 1999 a julho de 2000. Em cada amostragem foram coletadas 240 folhas, oriundas de 10 plantas, distribuídas ao acaso. As folhas foram retiradas em três estratos das copas das plantas (inferior, médio e superior), nos quadrantes norte, sul, leste e oeste. Nesses locais foram coletadas duas folhas, sendo uma "nova" outra madura. As amostras foram acondicionadas em sacos plásticos etiquetados e levadas ao laboratório para contagem dos ácaros, utilizando-se microscópio estereoscópico com aumento de 20 vezes.

Para avaliar a distribuição espacial do ácaro no erval foi determinada a variância e a média em cada data de amostragem. Com base nesses dados utilizou-se a Lei da Potência de Taylor $\left(\mathrm{S}^{2}=\mathrm{a} \cdot \mathrm{X}^{\mathrm{b}}\right)$ (Taylor 1961, Wilson et al. 1983, Ribeiro et al. 1990, Perruso \& Cassino 1997), que relaciona a variância com a média das densidades populacionais obtidas. Os coeficientes a (fator de amostragem) e b (índice de agregação da espécie) da Lei da Potência foram estimados pela análise da regressão da média e da variância. Para tal, a Lei de potência foi lineariazada resultando em: $\ln \left(\mathrm{S}^{2}\right)=\ln$ (a) + b. $\ln (\mathrm{X})$. Em seguida foi avaliada a relação entre a proporção de folhas infestadas com pelo menos um ácaro e o número médio de ácaro por folha, utilizando-se o modelo matemático proposto por Wilson \& Room (1983), representado pela equação 1 , onde $\mathrm{P}(\mathrm{I})$ é a proporção de folhas infestadas com o ácaro, X é o número médio de ácaro por folha, e a e b são os coeficientes de Taylor. A curva prevista pelo modelo matemático foi avaliada através da análise da regressão entre a proporção de folhas infestadas estimada e a proporção de folhas infestadas observadas no campo.

$$
P(I)=1-\exp \left(-\frac{\bar{X}}{a \bar{X}^{b-1}} \ln \left(a \bar{X}^{b-1}\right)\right)
$$

Equação 1

$\mathrm{O}$ número de amostras requeridas $\mathrm{n}$ para estimar a população do acarino foi determinado em função da densidade média a partir da equação 2 (Wilson et al. 1983). $n=\left(\frac{z \beta}{D}\right)^{2} \frac{P(O)}{P(I)}$

Equação 2

Neste caso, $\mathrm{D}$ corresponde à precisão admitida, sendo que neste trabalho foram escolhidos $\mathrm{D}=0,1$ e $\mathrm{D}=0,2$. $\mathrm{P}(\mathrm{O})$ é a probabilidade de não encontrar ácaros nas folhas, que está relacionada com $\mathrm{P}(\mathrm{I})$, pois $\mathrm{P}(\mathrm{O})=1-\mathrm{P}(\mathrm{I})$, e $\mathrm{z}_{\beta}$ é o valor da variável $z$, para o qual $Z(z)=2 \beta$, sendo $Z(z)$ a parte superior da distribuição normal padrão.

$$
Z(z)=\frac{1}{2}\left(1-\operatorname{erf}\left(\frac{z}{\sqrt{2}}\right)\right)
$$

Equação 3

Equação resultante em termos da função Erro (Equação 4).

$$
\operatorname{erf}(z)=\frac{2}{\sqrt{\pi}} \int_{0}^{z} \exp \left(-t^{2}\right) d t \quad \text { Equação } 4
$$

Neste trabalho foi utilizado $\beta=0,05$, o que implica em $z_{\beta}$ com o valor de 1,28155156 .

\section{Resultados e Discussão}

No período de avaliação do experimento $O$. yothersi ocorreu na densidade populacional média de 3,7 ácaros por folha, variando de 0,0 a 29,9, não incidindo em duas datas de amostragem.

A sua distribuição espacial foi agregada tendo base o valor $\mathrm{de} b=1,378$ (Fig. 1), que é significativamente maior que 1 pelo Teste t, conforme proposto por Silveira Neto et al. (1976), enquanto que o valor de $\mathrm{a}=12,962$ foi considerado elevado (Zalon et al. 1985, Wilson 1985).

A presença de predadores diminui a agregação de ácaros fitófagos (Wilson et al. 1984, Zalom 1985, Ribeiro et al. 1990). $\mathrm{Na}$ contagem dos ácaros $O$. yothersi a ocorrência de predadores foi inexpressiva, devendo ser reavaliado o padrão de distribuição da praga em locais com a presença freqüente de predadores.

Os valores dos pontos $\mathrm{P}(\mathrm{I})$ da curva, estimados pelo modelo

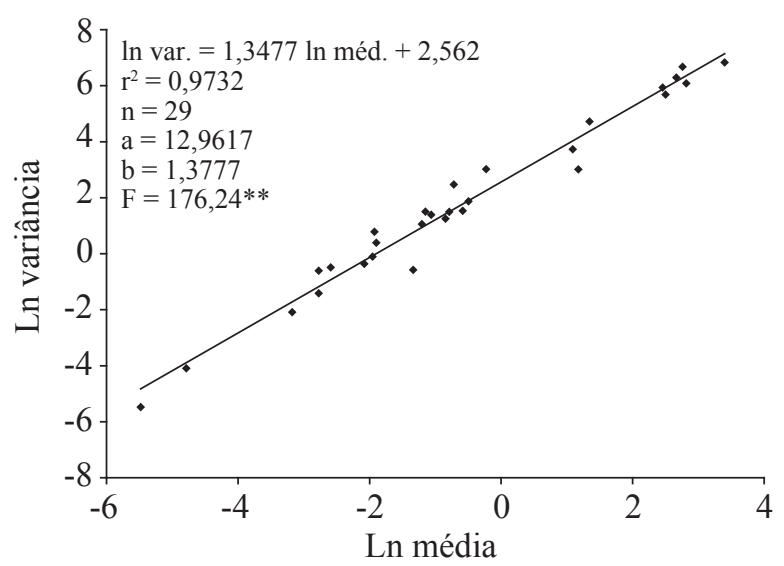

Fig. 1. Relação entre o logaritmo neperiano da variância e o da densidade média ( $\mathrm{n}^{\circ}$ de ácaros/folha) de O. yothersi em folhas de erva-mate I. paraguariensis, de abril de 1999 a julho de 2000, Cascavel, PR. 
proposto por Wilson \& Room (1983) com base no número médio de ácaro por folha e a relação variância/média, aumentou com o incremento da densidade média de ácaro por folha (Fig. 2) confirmando o comportamento agregado de $O$. yothers $i$ (Wilson 1985).

$\mathrm{O}$ ajuste do modelo utilizado avaliado pela análise da regressão entre a proporção de folhas infestadas estimadas e observadas (Fig. 3), explica 95\% da variação do modelo, indicando que a proporção de folhas infestadas estimadas pode ser usada como indicador do número médio de ácaros por folha.

O número de amostras requeridas para estimar a infestação do ácaro-roxo em função da sua densidade populacional média foliar, para o nível de precisão de $10 \%(\mathrm{D}=0,1)$, foi elevado nas densidades muito baixas (Fig. 4). No entanto, na medida em que o número de ácaro por folha aumentou o número de amostras necessárias foi reduzido, sendo que a partir de quatro ácaros o número de folhas a serem avaliadas foi de 204, chegando a 14 folhas com a infestação média de 30 ácaros por

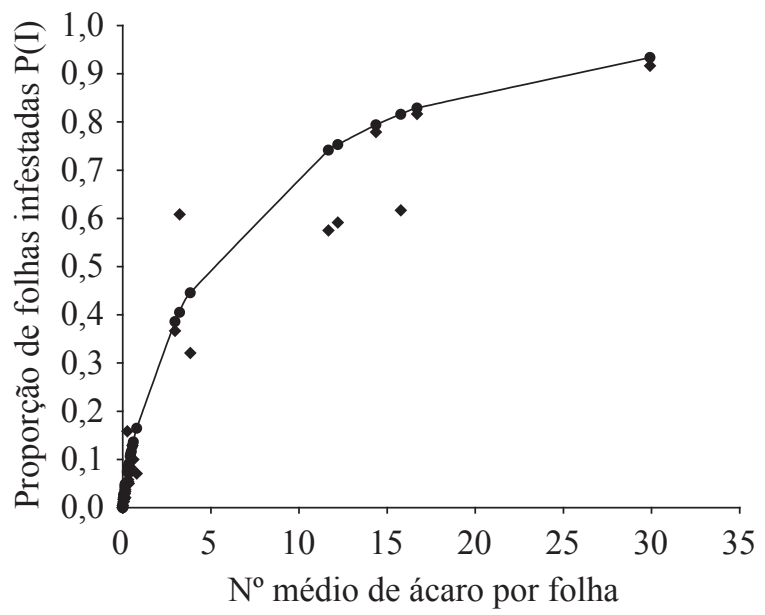

Fig. 2. Relação entre a proporção de folhas infestadas $\mathrm{P}(\mathrm{I})$ e o número médio de $O$. yothersi por folha em erva-mate $I$. paraguariensis, de abril de 1999 a julho de 2000, Cascavel, PR.

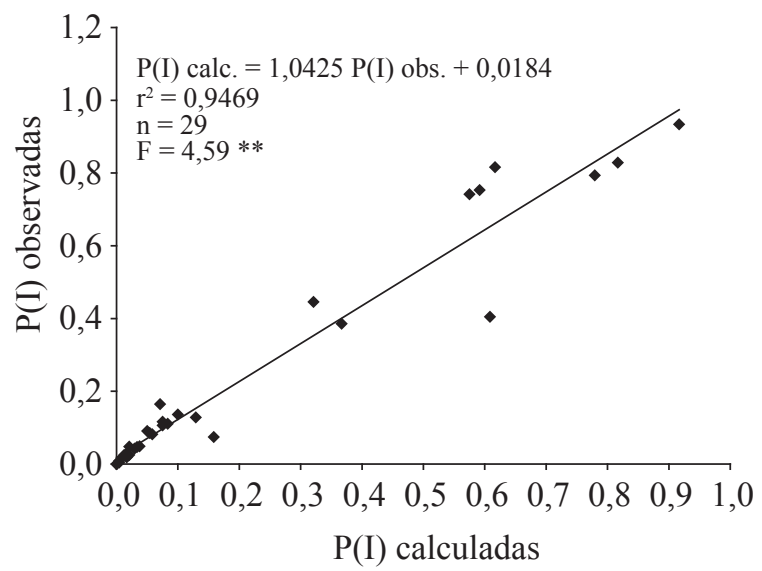

Fig. 3. Relação entre as proporções de folhas infestadas com $O$. yothersi calculadas pelo modelo matemático proposto por Wilson \& Room (1983) e as proporções de folhas infestadas observadas no erval, de abril de 1999 a julho de 2000, Cascavel, PR. folha (Tabela 1). São necessários, no entanto, novos estudos para determinar o nível de controle e para definir o tamanho do talhão ideal para a amostragem para a praga.

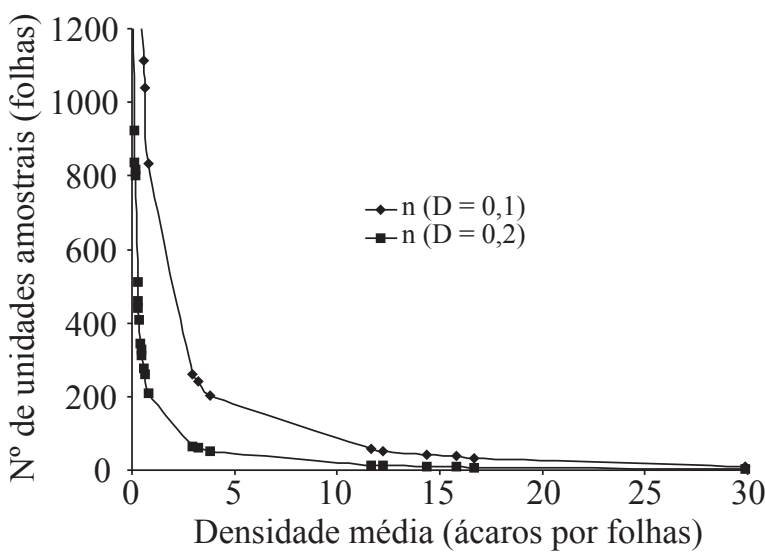

Fig. 4. Número de amostras requeridas em função da densidade média, e padrão de agregação de $O$. yothersi em ervamate I. paraguariensis, com nível de precisão $\mathrm{D}=0,1$ e $0,2 \mathrm{e}$ $\alpha=0,2\left(\mathrm{t}_{\alpha / 2}=1,28155156\right)$, de abril de 1999 a julho de 2000, Cascavel, PR.

Tabela 1. Número de amostras requeridas para estimar a infestação do ácaro O. yothersi em erva-mate(I. paraguariensis), em função da sua densidade média, e padrão de agregação, com nível de precisão $\mathrm{D}=0,1$ e 0,2 e $\alpha=0,2\left(\mathrm{t}_{\alpha / 2}=1,28155156\right)$, de abril de 1999 a julho de 2000, Cascavel, PR.

\begin{tabular}{lcc}
\hline \multirow{2}{*}{ Média } & \multicolumn{2}{c}{ Número de amostras requeridas } \\
\cline { 2 - 3 } & $\mathrm{D}=0,1$ & $\mathrm{D}=0,2$ \\
\hline 30 & 14 & 4 \\
25 & 20 & 5 \\
20 & 29 & 7 \\
18 & 35 & 9 \\
16 & 41 & 10 \\
14 & 50 & 12 \\
12 & 61 & 15 \\
10 & 76 & 19 \\
9 & 87 & 22 \\
8 & 99 & 25 \\
7 & 115 & 29 \\
6 & 136 & 34 \\
5 & 164 & 41 \\
4 & 204 & 51 \\
3 & 267 & 67 \\
2 & 383 & 96 \\
1 & 688 & 172 \\
0.5 & 1201 & 300 \\
\hline
\end{tabular}


O número de amostras requeridas para estimar a população do ácaro $O$. yothersi nos ervais pelo método presença-ausência, mesmo utilizando o nível de precisão de $\mathrm{D}=0,1$, apresenta grande potencial de aplicação prática.

O intervalo de tempo entre amostragens pode ser mantido o de quinze dias, uma vez que a duração média de um ciclo de ovo a adulto é de 17 dias para os machos e de 22 dias para as fêmeas (Alves et al. 2004).

\section{Agradecimentos}

Os autores agradecem ao CNPq pela concessão da bolsa de Produtividade em Pesquisa.

\section{Referências}

Alves, L.F.A., S. Spongoski, F.N.S. Vieira \& G.J. Moraes. 2004. Biologia e danos de Oligonychus yothersi (McGregor) (Acari: Tetranychidae) em Ilex paraguariensis. Arq. Inst. Biol. 71: 211-214.

Gouvea, A., L.C. Boaretto, C.F. Zanella \& L.F.A. Alves. 2006. Dinâmica populacional de ácaros (Acari) em plantas de ervamate (Ilex paraguariensis St. Hil.: Aquifoliaceae). Neotrop. Entomol. 35: 101-111.

Penteado, S.R.C. 1995. Principais pragas da erva-mate e medidas alternativas para o seu controle, p.109-120. In H. Winge, A.G. Ferreira, J.E.A. Mariath \& L.C. Tarasconi. (eds.), Erva-mate: Biologia e cultura no Cone Sul. Porto Alegre, Ed. UFRGS, $356 \mathrm{p}$.

Perruso, J.C. \& P.C.R. Cassino. 1997. Plano de amostragem presença-ausência para Selenaspidus articulatus (Morg.) (Homoptera: Diaspididae) na cultura de laranja. An. Soc. Entomol. Brasil 26: 321-326.
Silveira Neto, S., O. Nakano, D. Barbin \& N.A. Villa Nova. 1976. Manual de ecologia dos insetos. Piracicaba, Ceres, 419p.

Ribeiro, L.G., A. Villacorta \& L.A. Foerster. 1990. Plano de amostragem presença-ausência para Panonychus ulmi (Kock, 1836) (Acari: Tetranychidae) em macieira. An. Soc. Entomol. Brasil 19: 211-220.

Taylor, L.R. 1961. Aggregation, variance and the mean. Nature 189: 732-735.

Wilson, L.T. 1985. Estimating the abundance and impact of arthropod natural enemies on IPM systems, p.303-322. In M.A. Hoy \& D.C. Herzog, Biological control in agricultural IPM systems. New York, Academic Press, 555p.

Wilson, L.T., D. Gonzalez, T.F. Leigh, V. Magi, C. Foristiere \& P. Goodell. 1983. Within-plant distribution of spider mites (Acari: Tetranychidae) on cotton: A developing implementable monitoring program. Environ. Entomol. 12: 129-134.

Wilson, L.T., M.A. Hoy, F.G. Zalon \& J.M. Smilanick. 1984. Sampling mites in almonds: I- Within-tree distribution and clumping pattern of mites with comments on predator-prey interactions. Hilgardia 52: 1-3

Wilson, L.T. \& P.M. Room. 1983. Clumpling patterns of fruit and arthropods in cotton, with implications for binomial sampling. Environ. Entomol. 12: 50-54.

Zalom, F.G., C.E. Kennett, N.V. Connell, D. Flaherty, J.G. Morse \& L.T Wilson. 1985. Distribution of Panonychus ulmi (Mc Gregor) and Euseius tularensis Congdon on central Califórnia orange trees with implications for binomial sampling. Agric. Ecosyst. Environ. 14: 119-129.

Received 03/V/06. Accepted 28/XII/06. 